International Journal of Pure and Applied Mathematics

Volume 106 No. 4 2016, 1079-1086

ISSN: 1311-8080 (printed version); ISSN: 1314-3395 (on-line version)

url: http://www.ijpam.eu

doi: 10.12732/ijpam.v106i4.9

ijpam.eu

\title{
STRONGLY NILPOTENT RADICALS IN THE PROJECTIVE PRODUCT OF GAMMA RINGS AND THEIR INTRINSIC PROPERTIES
}

\author{
Ranu Paul \\ Department of Mathematics \\ Pandu College, Guwahati 781012 \\ Assam State, INDIA
}

\begin{abstract}
The object of this paper is to study strongly nilpotent radical in the projective product of gamma rings and their deep properties. This also includes some profound results on semi-prime Gamma-ring/prime Gamma-ring/semi-simple Gamma-ring. It is also shown that if the projective product of two Gamma-rings is semi-simple then the component Gamma-rings are also semi-simple and the converse of this result is also investigated.
\end{abstract}

AMS Subject Classification: 16N60, 16W25, 16U80

Key Words: strongly nilpotent ideal/strongly nilpotent radical/projective product of Gamma-rings

\section{Introduction}

In study of Gamma-ring theory which was introduced by Nobusawa [7] and later re-defined by Barnes [10], different kinds of radicals play an important role. There is a very strong theory of various radicals on general rings and Banach algebras $[1,6]$. Many prominent mathematicians have extended fruitfully many significant technical results on radicals of general ring to the radicals of Gammaring, see $[2,3,4,5,8,9,11]$.

Received: September 25, 2015

Published: March 8, 2016 (c) 2016 Academic Publications, Ltd.

url: www.acadpubl.eu 


\section{Fundamental Definitions}

The following concepts are useful for our purpose:

Definition 2.1. A gamma ring $\Gamma(X)$ in the sense of Nabusawa is said to be simple if for any two nonzero elements $x y \in X$, there exist $\Gamma \gamma \in$ such that $x \gamma y \neq 0$

Definition 2.2. A gamma ring $\Gamma(X)$ in the sense of Nabusawa is said to be semi-simple if for any nonzero elements $x \in X$, there exist $\Gamma \gamma \in$ such that $x \gamma x \neq 0$

Definition 2.3. If $I$ is an additive subgroup of a gamma ring $\Gamma(X)$ and $\Gamma X I \subseteq I$ (or $\Gamma I X \subseteq \mathrm{I}$ ), then $I$ is called a left (or right) gamma ideal of $X$. If $I$ is both left and right gamma ideal then it is said to be a gamma ideal of $\Gamma(X)$ or simply an ideal.

Definition 2.4. An ideal $I$ of a gamma ring $\Gamma(X)$ is said to be prime if for any two ideals $A$ and $B$ of $X, \Gamma A B \subseteq I=>A \subseteq I$ or $B \subseteq I$..

Definition 2.5. An ideal $I$ of the gamma $\operatorname{ring} \Gamma(X)$ is said to be semiprime if for any ideal $U$ of $X, \Gamma U U \subseteq I=>U \subseteq I$

Definition 2.6. An element $a$ of a gamma $\operatorname{ring} \Gamma(X)$ is strongly nilpotent if there exist a positive integer $n$ such that $Г Г Г Г Г ~(a)^{n} a=(a a a \ldots \ldots a) a=0$. A subset $S$ of $X$ is strongly nil if each of its elements is strongly nilpotent. $S$ is strongly nilpotent if there exist a positive integer $n$ such that $\Gamma(S)^{n} S=0$. Clearly a strongly nilpotent set is also strongly nil.

Definition 2.7. The strongly nilpotent radical, denoted by $S(X)$ of a gamma ring $\Gamma(X)$ is defined as the sum of all strongly nilpotent ideals of $\Gamma(X)$

Definition 2.8. Let $\Gamma\left(X_{1}, 1\right)$ and $\Gamma\left(X_{2}, 2\right)$ be two gamma rings. Let $X=X_{1} \times X_{2}$ and

$\Gamma \Gamma \Gamma={ }_{1} \times{ }_{2}$. Then defining addition and multiplication on $X$ and $\Gamma$ by,

$$
\begin{aligned}
& \left(x_{1}, x_{2}\right)+\left(y_{1}, y_{2}\right)=\left(x_{1}+y_{1} x_{2}+y_{2}\right), \\
& \left(\alpha_{1}, \alpha_{2}\right)+\left(\beta_{1}, \beta_{2}\right)=\left(\alpha_{1}+\beta_{1} \alpha_{2}+\beta_{2}\right)
\end{aligned}
$$

and $\left(x_{1}, x_{2}\right)\left(\alpha_{1}, \alpha_{2}\right)\left(y_{1}, y_{2}\right)=\left(x_{1} \alpha_{1} y_{1} x_{2} \alpha_{2} y_{2}\right)$ 
for every $\left(x_{1}, x_{2}\right)\left(y_{1}, y_{2}\right) \in X$ and $\Gamma\left(\alpha_{1}, \alpha_{2}\right)\left(\beta_{1}, \beta_{2}\right) \in$ $\Gamma(X)$ is a gamma ring. We call this gamma ring as the Projective product of gamma rings.

\section{Main Results}

Theorem 3.1. If $U$ is an ideal of $\Gamma(X)$, then $\Gamma U U$ is also an ideal of $\Gamma(X)$.

Proof. Since $U$ is an ideal of $\Gamma(X)$ so $\Gamma X U \subseteq U$ and $\Gamma U X \subseteq U$

Let $\Gamma V=U U$. Then $\Gamma \Gamma \Gamma U U \subseteq X U \subseteq U \subseteq X=>U U \subseteq X=>V \subseteq X$

Now, ГГГГГГГ $X V=X(U U)=(X U) U \subseteq U U=V=>X V \subseteq V$

Similarly, $\Gamma V X \subseteq V$ So $V$ is an ideal of $\Gamma(X)$

Theorem 3.2. If $\Gamma(X)$ is a prime gamma ring, then there does not exist any nonzero nilpotent ideal of $X$.

Proof. Let $\Gamma(X)$ be a prime gamma ring and $U$ be a strongly nilpotent ideal of $\Gamma(X)$

Then there exist a positive integer $n$ such that

$$
\begin{gathered}
\Gamma(U)^{n} U=0 \\
\Gamma \Gamma \Gamma=>(U U \ldots \ldots) U=0 \\
\Gamma \Gamma \Gamma \Gamma \Gamma=>(U U)(U U) \ldots(U U)=0 \\
\Gamma \Gamma \Gamma=>V V \ldots . . V=0, \text { where } V=U U \text { is an ideal of } X \\
\Gamma \Gamma \Gamma=>V(V \ldots . . . V)=0 \\
\Gamma \Gamma \Gamma=>V W=0, \text { where } W=V \ldots \text { is an ideal of } X \\
=>V=0 \text { or } W=0[\text { Since } \Gamma(X) \text { is prime }]
\end{gathered}
$$

If $W=0$, then by repeated application of prime-ness of $\Gamma(X)$, we get $V=0$

Thus in either case, $V=0$ which implies $U+U=0=>U=0$, since $\Gamma(X)$ is prime.

We assumed $U$ to be a strongly nilpotent ideal and obtained $U=0$ which implies that there does not exist any non zero strongly nilpotent ideal of a prime gamma ring. 
Theorem 3.3 If there does not exist any non zero strongly nilpotent ideal of a gamma ring $\Gamma(X)$, then it is a semi-prime gamma ring.

Proof. Let $\Gamma(X)$ be gamma ring in which there does not exist any non zero strongly nilpotent ideal and let $U$ be an ideal of $\Gamma(X)$ such that,

$$
\Gamma \Gamma U U=0=>(U)^{n} U=0, n=1
$$

$=>U$ is a strongly nilpotent ideal $=>U=0$

Thus we get, $\Gamma U U=0=>U$. Hence $\Gamma(X)$ is a semi-prime gamma ring.

Theorem 3.4 Every prime gamma ring is semi-simple.

Proof. Let $\Gamma(X$,$) be a prime gamma ring.$

Since a prime gamma ring does not contain any non zero strongly nilpotent ideal so the only strongly nilpotent ideal is the zero ideal. $S(X)$ being the sum of all strongly nilpotent ideals of $\Gamma(X)$ is equal to zero. i.e $S(X)=0$

Again $S(X)=0$ if and only if $X$ is semi-simple [11]

Hence $\Gamma(X$,$) is semi-simple.$

Theorem 3.5 Every simple gamma ring is a prime gamma ring.

Proof. Let $\Gamma(X$,$) be a simple gamma ring. Then for any two nonzero$ elements $x y \in X$, there exist $\Gamma \gamma \in$ such that $x \gamma y \neq 0$

Let $U, V$ be two ideals of $X$ such that $\Gamma U V=0$. We show $U=0$ or $V=0$

If possible let $U \neq 0$ and $V \neq 0$. Then there exist $0 \neq x \in U$ and $0 \neq x \in V$

Since $\Gamma(X$,$) is simple so there exist \Gamma \gamma \in$ such that $x \gamma y \neq 0$

Now $\Gamma x \gamma y \in U V=0=>x \gamma y=0$, which is a contradiction. So our supposition is wrong.

So we must have $U=0$ or $V=0$. Hence $\Gamma(X$,$) is a prime gamma ring.$

Theorem 3.6 If $\Gamma(X$,$) be a gamma ring then S(X)=0$ implies $X$ is semi-prime.

Proof. If $S(X)=0$ then $X$ is semi-simple [11]. Then for any nonzero element $x \in X$, there exist $\Gamma \gamma \in$ such that $x \gamma x \neq 0$. We show $X$ is semi-prime.

Let $U$ be an ideal of $X$ such that $\Gamma U U=0$. We show $U=0$

If possible let $U \neq 0$. Then there exist $0 \neq x \in U$. Since $\Gamma(X$,$) is semi-$ simple so there exist $\Gamma \gamma \in$ such that $x \gamma x \neq 0$

But $\Gamma x \gamma x \in U U=0=>x \gamma x=0$, which is a contradiction. So our supposition is wrong. 
So we must have $U=0$. Hence $\Gamma(X$,$) is a semi-prime gamma ring and the$ result.

Theorem 3.7 Let $\Gamma\left(X_{1}, 1\right)$ and $\Gamma\left(X_{2}, 2\right)$ be two gamma rings and $\Gamma(X)$ be their Projective product with the right operator rings $R_{1}, R_{2}$ and $R$ respectively. Then $R=R_{1} \times R_{2}$, where $R_{1} \times R_{2}$ is defined as, for any $t=\left(t_{1}, t_{2}\right) \in R_{1} \times R_{2}$ and $m=(x, y) \in X, m t=(x, y)\left(t_{1}, t_{2}\right)=\left(x t_{1}, y t_{2}\right)$

Proof. Since $R_{1}, R_{2}$ are rings so with this definition $R$ is also a ring. and

We have, $\Gamma R=\left\{\sum_{i}\left[\gamma_{i}, z_{i}\right]: \gamma_{i} \in, z_{i} \in X, \Gamma R_{1}=\left\{\sum_{i}\left[\alpha_{i}, x_{i}\right]: \alpha_{i} \in{ }_{1}, x_{i} \in X_{1}\right.\right.$

$$
\Gamma R_{2}=\left\{\sum_{i}\left[\beta_{i}, y_{i}\right]: \beta_{i} \in{ }_{2}, y_{i} \in X_{2}\right.
$$

Let $t=\sum_{i}\left[\gamma_{i}, z_{i}\right] \in R$ and $m=(x, y) \in X$ be any elements, where $\Gamma \gamma_{i}=$ $\left(\alpha_{i}, \beta_{i}\right) \in$ and $z_{i}=\left(x_{i} y_{i}\right) \in X$

Now, $m t=m \sum_{i}\left[\gamma_{i}, z_{i}\right]=\sum_{i} m \gamma_{i} z_{i}$

$$
\begin{gathered}
=\sum_{i}(x, y)\left(\alpha_{i}, \beta_{i}\right)\left(x_{i}, y_{i}\right)=\sum_{i}\left(x \alpha_{i} x_{i}, y \beta_{i} y_{i}\right)=\left(\sum_{i} x \alpha_{i} x_{i} \sum_{i} y \beta_{i} y_{i}\right) \\
=\left(x \sum_{i}\left[\alpha_{i}, x_{i}\right], y \sum_{i}\left[\beta_{i}, y_{i}\right]\right)=(x y)\left(\sum_{i}\left[\alpha_{i}, x_{i}\right] \sum_{i}\left[\beta_{i}, y_{i}\right]\right) \\
=>m t=m\left(\sum_{i}\left[\alpha_{i}, x_{i}\right] \sum_{i}\left[\beta_{i}, y_{i}\right]\right)
\end{gathered}
$$

So $R=R_{1} \times R_{2}$, which is the right operator ring $\Gamma(X)$

Theorem 3.8 Let $\Gamma\left(X_{1}, 1\right)$ and $\Gamma\left(X_{2}, 2\right)$ be two gamma rings and $\Gamma(X)$ be their Projective product. Then every strongly nilpotent ideal of $\Gamma(X)$ gives rise to two strongly nilpotent ideals of $\Gamma\left(X_{1}, 1\right)$ and $\Gamma\left(X_{2}, 2\right)$ and vice versa.

Proof. Let $U$ be a strongly nilpotent ideal of $\Gamma(X)$. Since $U$ is an ideal $X$ so $U=A \times B$, where $A, B$ are ideals of $X_{1}$ and $X_{2}$ respectively.

Then there exist a positive integer $n$ such that $\Gamma(U)^{n} U=0$

$$
\Gamma \Gamma \Gamma=>(U U \ldots . . U) U=0
$$

$$
\begin{gathered}
\text { ГГГГГГ }=>((A \times B)(1 \times 2)(A \times B)(1 \times 2) \ldots .(A \times B)(1 \times 2))(A \times B)=0 \\
\Gamma Г Г Г Г \Gamma=>(A \times B)(1 \times 2)(A \times B)(1 \times 2) \ldots .(A \times B)(1 \times 2)=0
\end{gathered}
$$




$$
\begin{aligned}
& \text { ГГГГГГ }=>\left(A_{1} A_{1} \ldots ._{1} A\right) \times\left(B_{2} B_{2} \ldots ._{2} B\right)=0 \\
& \text { ГГГГГГ }=>\left(\left(A_{1} A_{1} \ldots A_{1}\right) A\right) \times\left(\left(B_{2} B_{2} \ldots . B_{2}\right) B\right)=0 \\
& \Gamma \Gamma=>\left(\left(A_{1}\right)^{n} A\right) \times\left(\left(B_{2}\right)^{n} B\right)=0 \\
& \Gamma \Gamma=>\left(A_{1}\right)^{n} A=0 \text { and }\left(B_{2}\right)^{n} B=0
\end{aligned}
$$

$=>A$ and $B$ are strongly nilpotent ideals of $X_{1}$ and $X_{2}$ respectively.

Conversely, let $A$ and $B$ be two strongly nilpotent ideals of $X_{1}$ and $X_{2}$ respectively.

So $U=A \times B$ is an ideal of $\Gamma(X)$.

Since $A$ and $B$ are strongly nilpotent ideals so there exist positive integers $m n$ such that $\Gamma \Gamma\left(A_{1}\right)^{m} A=0$ and $\left(B_{2}\right)^{n} B=0$

Without the loss of generality let, $m \leq n$ Then we have,

$$
\begin{gathered}
\Gamma \Gamma \Gamma(U)^{n} U=\left(\left(A_{1}\right)^{n} A\right) \times\left(\left(B_{2}\right)^{n} B\right) \\
\Gamma \Gamma \Gamma=\left(\left(A_{1}\right)^{m}\left(A_{1}\right)^{n-m} A\right) \times\left(\left(B_{2}\right)^{n} B\right) \\
\Gamma \Gamma \Gamma=\left(\left(A_{1}\right)^{m} A\right)\left(\left({ }_{1} A\right)^{n-m}\right) \times\left(\left(B_{2}\right)^{n} B\right)
\end{gathered}
$$

$=0 \times 0=0[\mathrm{Using}(1)]$

Thus there exists a positive integer $n$ such that $\Gamma(U)^{n} U=0$. So $U$ is also strongly nilpotent and hence the theorem.

Theorem 3.9 Let $\Gamma\left(X_{1},{ }_{1}\right)$ and $\Gamma\left(X_{2}, 2\right)$ be two gamma rings and $\Gamma(X)$ be their Projective product. Then $S(X)=S\left(X_{1}\right) \times S\left(X_{2}\right)$

Proof. $S(X)=$ Sum of all strongly nilpotent ideals of $X$

$$
\begin{aligned}
& =>S(X)=\sum_{P \text { is a strongly }} P \\
& \text { nilpotent ideal of } X \\
& =>S(X)=\sum \quad(A \times B)[\text { By theorem } \\
& A \text { and } B \text { are strongly } \\
& \text { nilpotent ideals of } X_{1} \text { and } X_{2} \text { respectively }
\end{aligned}
$$

$$
=S(X)=\left(\sum_{\begin{array}{l}
\text { Ais a strongly } \\
\text { nilpotent ideal of } X_{1}
\end{array}} A\right) \times\left(\begin{array}{l}
\sum_{\text {Bis a strongly }}^{\text {nilpotentideal of } X_{2}} \\
\text { nilpon }
\end{array}\right)
$$


$=>S(X)=S\left(X_{1}\right) \times S\left(X_{2}\right)$. Hence the result.

Theorem 3.10 If the projective product of two gamma rings is semi-simple then the component gamma rings are also semi-simple and vice versa.

Proof. Let $\Gamma\left(X_{1}, 1\right)$ and $\Gamma\left(X_{2}, 2\right)$ be two gamma rings and $\Gamma(X)$ be their Projective product. Suppose $\Gamma(X)$ is semi-simple. We show $\Gamma\left(X_{1},{ }_{1}\right)$ and $\Gamma\left(X_{2}, 2\right)$ are also semi-simple.

Since $\Gamma(X)$ is semi-simple, so the strongly nilpotent radical is zero i.e $S(X)=0$

By our theorem 3.9, $S(X)=S\left(X_{1}\right) \times S\left(X_{2}\right)$

So we have, $S\left(X_{1}\right) \times S\left(X_{2}\right)=0$

$=>S\left(X_{1}\right)=0$ and $S\left(X_{2}\right)=0$ [Since $A \times B=0=>A=0, B=0$ ]

$=>$ Both $X_{1}$ and $X_{2}$ are semi-simple.

Conversely, let Both $X_{1}$ and $X_{2}$ be semi-simple. We show $X$ is also semisimple.

For this, let, $z=(x, y) \in X$ be any element such that $\Gamma z \gamma z=0 \forall \gamma \in$, where $\gamma=(\alpha \beta)$

$$
\begin{gathered}
\Gamma z \gamma z=0 \forall \gamma \in \\
\Gamma \Gamma=>(x \alpha x, y \beta y)=0 \forall \alpha \in_{1}, \beta \in_{2} \\
\Gamma \Gamma=>x \alpha x=0 \text { and } y \beta y=0 \forall \alpha \in_{1}, \beta \in_{2}
\end{gathered}
$$

$=>x=0$ and $y=0\left[X_{1}\right.$ and $X_{2}$ are semi-simple $]$

$$
=>z=(x, y)=(0,0)=0
$$

So, $X$ is also semi-simple and hence the result.

\section{References}

[1] C.E. Rickart, General theory of Banach algebra, D. Von Nostrand Company, INC Princetor, New Jersey, Toronto, London, New York.

[2] G.L. Booth, Radicals of matrix gamma rings, Math. Japonica 33, No.3 (1988), 325-334

[3] G.L. Booth, On the radicals of $\Gamma_{N}$-rings, Math. Japan 32, No.3(1987), 357-372.

[4] G.L. Booth, Super nilpotent radicals of $\Gamma$-rings, Acta Math. Hungar. 54, No.3-4(1989), 201-208.

[5] G.L. Booth, Characterizing the nil radical of a gamma ring, Quaestiones Math., Vol 9, Nos 1-4, 1986, pp 55-67.

[6] K.R. Goodear, Ring Theory, (Marcel Dekker) 1976.

[7] N. Nobusawa, On a Generalisation of the Ring Theory, Osaka J. Math., 1(1964), 81- 89. 
[8] N. Nobusawa, A contribution to the radical theory of gamma rings, Ph.D. thesis, University of Stellenbosch, 1985.

[9] N.J. Groenewald, Radicals of Г-rings, Quaestiones Math. 7, No.4(1984), 337-344.

[10] W.E. Barnes, On the $\Gamma$-rings of Nobusawa, Pacific Journal of Math. 18, No.3(1966), 411-422.

[11] W. E. Coppage and J. Luh, Radicals of gamma rings, J. Math. Soc. Japan 23 (1971), 41-52.

[12] Y.C. Guo, Relations among radicals of $\Gamma$-rings and a result of Kyuno, J. Math. Res. Exposition (English Ed) N0.1(1986), 31-34. 